\title{
Cerebral blood flow identifies responders to transcranial magnetic stimulation in auditory verbal hallucinations
}

\author{
P Homan ${ }^{1,3}$, J Kindler ${ }^{1,3}$, M Hauf $^{2}$, D Hubl ${ }^{1}$ and T Dierks ${ }^{1}$
}

Auditory hallucinations comprise a critical domain of psychopathology in schizophrenia. Repetitive transcranial magnetic stimulation (TMS) has shown promise as an intervention with both positive and negative reports. The aim of this study was to test resting-brain perfusion before treatment as a possible biological marker of response to repetitive TMS. Twenty-four medicated patients underwent resting-brain perfusion magnetic resonance imaging with arterial spin labeling (ASL) before 10 days of repetitive TMS treatment. Response was defined as a reduction in the hallucination change scale of at least $50 \%$. Responders $(n=9)$ were robustly differentiated from nonresponders $(n=15)$ to repetitive TMS by the higher regional cerebral blood flow (CBF) in the left superior temporal gyrus (STG) $(P<0.05$, corrected) before treatment. Resting-brain perfusion in the left STG predicted the response to repetitive TMS in this study sample, suggesting this parameter as a possible bio-marker of response in patients with schizophrenia and auditory hallucinations. Being noninvasive and relatively easy to use, resting perfusion measurement before treatment might be a clinically relevant way to identify possible responders and nonresponders to repetitive TMS.

Translational Psychiatry (2012) 2, e189; doi:10.1038/tp.2012.114; published online 20 November 2012

\section{Introduction}

Hallucinations are perceptual experiences in the absence of an adequate external stimulus, and auditory verbal hallucinations (AVH) are a key symptom of psychosis. In schizophrenia, the 1-month prevalence of these hallucinations exceeds $70 \%,{ }^{1}$ and in $25-30 \%$ of patients these perceptions are resistant to medication, resulting in functional disability and a low quality of life. ${ }^{2,3}$ With limited therapeutic options, the development of new treatment strategies ${ }^{4}$ is needed. One such intervention is repetitive transcranial magnetic stimulation (TMS). Hoffman was the first to report that low-frequency TMS decreased the severity of medication-resistant AVH when the coil is directed at the left temporoparietal ${ }^{5}$ area. Since that initial report, a number of studies have been conducted with a similar treatment paradigm and focus. ${ }^{6-21}$ Nevertheless, in a recent study with the largest sample size so far, no difference between sham and stimulation has been found. ${ }^{22}$ However, it has been suggested that patients respond differentially to $\mathrm{TMS},{ }^{23}$ making the exploration for possible predictors desirable. Predictors may be either found in the clinical phenomenology of symptoms or in brain function. With respect to symptoms, only minimal response to TMS has been reported in continuous hallucinators. ${ }^{6,14}$ With regard to brain function, only functional magnetic imaging (fMRI), but not resting perfusion, has been used to predict response to TMS in AVH. Using temporoparietal TMS, lower levels of hallucination-related activation in Broca's area predicted the response rate in intermittent hallucinators, and reduced coupling between Wernicke's and a right homolog of Broca's area predicted the response-rate in continuous hallucinators. ${ }^{15} \mathrm{fMRI}$ requires detecting the symptoms of hallucinations in the scanner, which is extremely challenging depending on many uncontrollable factors, thus making it clinically practical only in a few patients. Therefore, predictors, such as resting neuronal activity, not requiring symptom and patient cooperation, would clearly be clinically more useful. We recently showed that the improvement of symptoms after 10 days of TMS treatment was associated with decreased neuronal activity in the primary auditory cortex, Broca's area and cingulate gyrus, ${ }^{24}$ suggesting that resting perfusion, reflecting neuronal activity, is an appropriate marker for the effectiveness of TMS. In the current study, we applied a different approach. Our aim was to identify responders and nonresponders post hoc using resting-state perfusion and not to test the effectiveness of TMS itself. Considering that there is a hyperactivity in language-related regions in AVH patients, which is related to symptom severity, ${ }^{25-27}$ we postulated that cerebral-resting perfusion before TMS treatment is higher in responders compared with nonresponders. To achieve a larger sample size, we here pooled patients using two different TMS protocols. A repetitive TMS protocol that has recently been introduced into clinical research is theta-burst stimulation. ${ }^{28-30}$ Compared with the often-used $1-\mathrm{Hz}$ stimulation protocol, theta-burst stimulation has the advantage that the application duration is very short, making the treatment

\footnotetext{
${ }^{1}$ Department of Psychiatric Neurophysiology, University Hospital of Psychiatry, University of Bern, Bern, Switzerland and ${ }^{2}$ SCAN, Institute of Diagnostic and Interventional Neuroradiology, Inselspital, University of Bern, Bern, Switzerland

Correspondence: Professor T Dierks, Department of Psychiatric Neurophysiology, University Hospital of Psychiatry, Bolligenstrasse 111, CH-3000 Bern 60, Switzerland. E-mail: dierks@puk.unibe.ch

${ }^{3}$ These authors contributed equally to this work.

Keywords: arterial spin labeling; auditory verbal hallucinations; cerebral blood flow; schizophrenia; superior temporal gyrus; transcranial magnetic stimulation Received 16 August 2012; revised 12 September 2012; accepted 7 October 2012
} 
more convenient for the patient. In a recent study, we found theta-burst and $1-\mathrm{Hz}$ TMS to be equally effective, ${ }^{24}$ and therefore we randomly assigned patients to receive either a 1$\mathrm{Hz}$ or theta-burst TMS protocol. Before treatment, we measured whole-brain resting perfusion using magnetic resonance (MR) arterial spin labeling (ASL), an MR technique that provides a direct quantitative measure of cerebral blood flow (CBF). ${ }^{31-33}$ ASL is a noninvasive technique that reliably measures whole-brain CBF with converging results to those obtained with invasive PET(Positron emission tomography) perfusion imaging. ${ }^{34}$

\section{Materials and methods}

Patients and clinical investigation. Inclusion criteria were the diagnosis of schizophrenia or schizoaffective disorder according to ICD-10 (F20, F25), age between 18 and 65 years, right-handedness and medication-resistant $\mathrm{AVH}$. Therapy refractoriness was defined as having no response to at least two different antipsychotic treatment options within licensed dose ranges, each administered for at least 8 weeks. Medication dose remained stable during the treatment. Exclusion criteria were history of epileptic seizures, signs of elevated neuronal activity by electroencephalography, MR contraindications and medical disorders other than schizophrenia or schizoaffective disorder. Substance misuse in the 4 weeks before treatment was ruled out by a urinary drug screen before treatment. All patients underwent identical diagnostic procedures and identical MR protocols. The diagnostic procedure was performed on the basis of clinical interviews and psychiatric history. The assessment of psychopathology, consisting of the auditory hallucination rating scale (AHRS), ${ }^{35}$ the hallucination change scale ${ }^{35}$ and the positive and negative symptom scale (PANSS), ${ }^{36}$ was performed before and after 10 days of TMS treatment. To detect clinically relevant improvements in symptoms, we used a conservative level of at least $50 \%$ in the hallucination change scale to define response to TMS, similar to the one used in previous studies. ${ }^{14,35}$ The Edinburgh handedness scale $^{37}$ was assessed to ensure that all patients were righthanded. The investigation was conducted in accordance with the Declaration of Helsinki and approved by the local ethics committee. Patients provided informed written consent to participate in the study.

Experimental design. MRI was conducted on a 3-T wholebody MRI system (Magnetom Trio, Siemens Medical Systems, Erlangen, Germany) with a standard 12-channel radiofrequency head coil. High-resolution three-dimensional structural (s)MRI and ASL were acquired before TMS. T1weighted three-dimensional magnetization-prepared rapid gradient echo scans were recorded (number of slices $=176$, matrix $=256 \times 256$, slice thickness $=1 \mathrm{~mm}$, voxel size $=1$ $\times 1 \times 1 \mathrm{~mm}^{3}$ ), serving as a high-resolution three-dimensional anatomical template for coregistration with functional data. A pseudo-continuous ASL technique was used to measure $\mathrm{CBF}{ }^{38}$ In this gradient-echo echo-planar imaging sequence, interleaved images with and without labeling were acquired. A delay of $1250 \mathrm{~ms}$ was applied between the end of the labeling pulse (label time $=1720 \mathrm{~ms}$ ) and image acquisition (slice acquisition time $=45 \mathrm{~ms}$ ), in order to reduce transit artifact (field of view $=220 \mathrm{~mm}^{2}$, matrix $=64 \times 64$, repetition time/echo time $=4000 / 18 \mathrm{~ms}$ and flip angle $=25^{\circ}$ ). A total of 14 slices (voxel size $=3.4 \times 3.4 \times 6 \mathrm{~mm}^{3}, \quad$ ga $P=1.5 \mathrm{~mm}$ ) were acquired in the anterior and posterior commissure line, from inferior to superior, in a sequential order. The pseudocontinuous ASL scan comprised 80 acquisitions. ASL data analysis was performed using $\mathrm{aslm}^{39}$ (downloadable at http://aslm.sourceforge.net), a newly developed toolbox that allows multiple subjects to be analyzed at once and to perform region of interest analysis of ASL data. aslm is an object-oriented toolbox based on Matlab (Matlab version 8, release 14; The MathWorks, Natick, MA, USA), SPM 8 (Wellcome Department of Imaging Neuroscience, London, England; http://www.fil.ion.ucl.ac.uk/spm/software/spm8/) and marsbar. ${ }^{40}$ All ASL time series were first realigned to correct for motion artifacts. We calculated flow-time series by subtracting the labeling images from the control images, and subsequently computed mean CBF images for each subject. ${ }^{41}$ Each individual subject's T1 anatomy was segmented into gray matter (GM) and white matter. Mean ASL images were then coregistered to GM-segmented T1. T1, GM, white matter and ASL images were normalized to the SPM MNI T1 template. ASL images were spatially smoothed with a three-dimensional 8-mm Full Width at Half Maximum Gaussian kernel. Data were $z$-transformed ( $z=$ (voxel CBF-global GM CBF)/s.d.) to remove sources of variance caused by differences of global mean CBF between acquisitions and corrected for GM using GM segments as inclusive masks. For visualization, CBF maps and clusters of the whole-brain analysis were overlaid onto T1-weighted MR image slices using aslm.

Neuronavigated TMS. We chose area Spt (Sylvian parietotemporal), located in the sylvian fissure at the parietotemporal boundary, as the target region for TMS. Area Spt is a sensorimotor interface between the sensory and motor speech systems, ${ }^{42}$ is strongly left dominant, ${ }^{43}$ and is activated equally by the perception and reproduction of aurally or visually presented words. There is evidence of functional abnormalities within area Spt in patients with schizophrenia. ${ }^{44}$ Because area Spt is connected to the frontal and temporal language regions that are involved in the generation of $\mathrm{AVH}$, it qualifies as an ideal target region for TMS modulation of $\mathrm{AVH}$. A modified version of an evaluated language-processing task introduced by Hickok et al. ${ }^{43}$ was used to localize the target region (Spt) for TMS treatment in an $\mathrm{fMRI}$ scan of each patient in the first MR measurement. This measurement took place immediately after the resting perfusion measurement with ASL. After superimposing the individual's $\mathrm{fMRI}$ activation map on its anatomical mesh (three dimensional reconstruction of T1 image), a TMS target point was created at area Spt. A custom TMS stimulator (MagPro R 100, Medtronic Functional Diagnostics, Skovlunde, Denmark) was used to generate repetitive biphasic magnetic pulses. Magnetic pulses were delivered with a figure-8 coil (Magnetic Coil Transducer MC-B70, Medtronic). Stimulation of the motor cortex with single TMS pulses until a movement of the contralateral thumb was detected permitted the identification of the individual resting motor thresholds. ${ }^{45}$ The center of the coil was held tangentially to the skull. TMS 
Table 1 Patient characteristics with mean values, s.d., statistical differences with $P$-values

\begin{tabular}{|c|c|c|c|c|c|c|}
\hline Characteristic & Responders $(\mathrm{n}=9)$ & Non-responders $(\mathrm{n}=15)$ & Test & Value & $d f$ & $P$ \\
\hline Gender, no. F/M & $4 / 5$ & $10 / 5$ & Fisher's exact & NA & NA & 0.4 \\
\hline Diagnosis, no. F20/F25 & $7 / 2$ & $14 / 1$ & Fisher's exact & NA & NA & 0.5 \\
\hline Age, mean (s.d.), years & $38.3(13.9)$ & $44.4(10.1)$ & $t$-test & 1.3 & 22 & 0.2 \\
\hline Age at onset, mean (s.d.), years & $24.7(6.5)$ & $26.6(6.6)$ & $t$-test & -1.4 & 22 & 0.2 \\
\hline Duration of disease, mean (s.d.), years & $12.9(7.1)$ & $18.6(10.6)$ & $t$-test & -1.2 & 22 & 0.2 \\
\hline Chlorpromazine equivalent dose at study entry, mean (s.d.) & $448.9(132.2)$ & $586.2(257.1)$ & $t$-test & -1.5 & 22 & 0.1 \\
\hline PANSS score before TMS, mean (s.d.) & $75.8(17.7)$ & $72.6(15.4)$ & $t$-test & 0.5 & 22 & 0.6 \\
\hline AHRS score before TMS, mean (s.d.) & $33(6.7)$ & $35.7(6.1)$ & $t$-test & 1.0 & 22 & 0.3 \\
\hline Global mean CBF, corrected for $\mathrm{GM}\left(\mathrm{ml} 100 \mathrm{~g}^{-1} \mathrm{~min}^{-1}\right)$ & $58.3(12.5)$ & $52.0(9.0)$ & $t$-test & 1.5 & 22 & 0.2 \\
\hline
\end{tabular}

Abbreviations: AHRS, auditory hallucination rating scale; CBF, cerebral blood flow; df, degrees of freedom; F, female; F20, schizophrenia; F25, schizoaffective disorder (according to ICD-10); GM, gray matter; M, male; NA, not applicable; PANSS, positive and negative syndrom scale; TMS, transcranial magnetic stimulation.

pulse intensity was adjusted to $90 \%$ of the resting motor threshold. Patients were randomly assigned to receive a 1$\mathrm{Hz}(n=12)$ or theta-burst $(n=12)$ TMS protocol. In both treatment groups, the target area was stimulated for 10 consecutive days. Stimulation at $1 \mathrm{~Hz}$ was applied once a day, according to the protocol used by Hoffman et al. ${ }^{35}$ In the theta-burst group, each burst contained three pulses at $30 \mathrm{~Hz}$, repeated with an interburst interval of $100 \mathrm{~ms}^{29}$ Theta burst was applied in double trains with a 15-min intertrain interval. On the first 3 days, 2 double trains of theta burst were applied (based on the protocol in Nyffeler et al. ${ }^{29}$ ), whereas on days 3-10 one double-theta-burst train was applied. Safety protocols were in accordance with international safety standards of TMS experimentation. ${ }^{46} \mathrm{~A}$ frameless, ultrasound-based stereotactic system was used for neuronavigation (BrainvoyagerTMS Neuronavigator System, Brain Innovation, BV 2006, ${ }^{47}$ Maastricht, The Netherlands). After superimposing the individual's $\mathrm{AMRI}$ activation map on its anatomical mesh (three-dimensional reconstruction of T1 image), a TMS target point was created at area Spt. Details on the fMRI stimulation paradigm have recently been published elsewhere. ${ }^{24}$

Statistical analysis: CBF. Average mean GM CBF values of responders and nonresponders were compared in a twosample $t$-test. A global and assumption-free second-level random-effect analysis was then performed using a twosample $t$-test on GM CBF images obtained in each participant (degree of freedom $(\mathrm{df})=22$ ). We report clusters with a voxel-level threshold of $P<0.001$ (uncorrected) at a cluster-level threshold of $P<0.05$, whole-brain corrected for familywise error.

\section{Results}

Clinical data. Twenty-four patients were included in the study. Clinical and demographic characteristics of the subject sample are detailed in Table 1. After 10 days of TMS treatment, patients were rated again by a blinded clinician. Nine patients showed a clinical improvement of at least $50 \%$ and were therefore classified as responders; the other 15 patients were classified as nonresponders. Responders and nonresponders did not differ in the levels of AHRS and PANSS before treatment (Table 1). In addition, the frequency item $(P=0.8)$, the reality item $(P=0.4)$ and the loudness item $(P=0.1)$ of the AHRS did not differ in responders and nonresponders. Responders and nonresponders were not different in age, gender, age of onset, disease duration or medication levels (Table 1). No differences were detected between the $1-\mathrm{Hz}$ (mean: $0.32 \pm 0.39$ ) and theta-burst (mean: $0.32 \pm 0.32$ ) protocols comparing the amount of improvement as measured with the hallucination change scale $(P=1)$. There was no difference in the relationships of responders to nonresponders in the $1-\mathrm{Hz}$ group (4:8) and in the theta-burst group $(5: 7, P=1)$.

ASL data. The average mean global CBF in the responders and nonresponders was $58.3( \pm 12.5)$ and 52.0 $( \pm 9.0) \mathrm{ml} 100 \mathrm{~g}^{-1} \mathrm{~min}^{-1}$, respectively (Table 1$)$. The average mean global CBF images of responders and nonresponders are shown in Figure 1. As average mean global CBF did not differ between responders and nonresponders $(P=0.2)$, analyses were performed with normalized data to reduce variability introduced by nonspecific fluctuations in global CBF. In the whole-brain random-effect analysis, two contrasts were tested: first, responders $>$ nonresponders, that is, voxels that were significantly higher perfused in responders than in nonresponders before treatment, and second the inverse contrast (nonresponders $>$ responders). In the first contrast, one single cluster of 120 voxels survived a cluster-level whole-brain correction for familywise error in the left superior temporal gyrus (STG) (Brodmann area 22, peak: $t[22]=7.28$, $x / y / z=-58 /-26 / 4$, Figure 2). No clusters survived in the inverse contrast (nonresponders $>$ responders).

ASL data and psychopathology. The normalized levels of CBF in the left STG before treatment were correlated (Spearman's rank correlation) with the amount of improvement, as measured with the hallucination change scale ( $r=0.7$, df: $22, P<0.0001$, Figure 3 ). There was no relationship between the level of perfusion in STG in responders and nonresponders and the levels of symptoms as measured by the PANSS ( $r=-0.04$, df: 22, $P=0.9$ ) before treatment. In addition, pretreatment scores of the AHRS items frequency $(P=0.9)$, reality $(P=0.7)$ and loudness $(P=0.5)$ were not correlated with the level of STG perfusion.

Reciever operator characteristic (ROC) analysis. To further investigate the discriminative power of CBF in the left STG, we conducted a receiver operator characteristic (ROC) analysis. That way, we measured how well CBF in the left STG could be used to distinguish between responders 
a
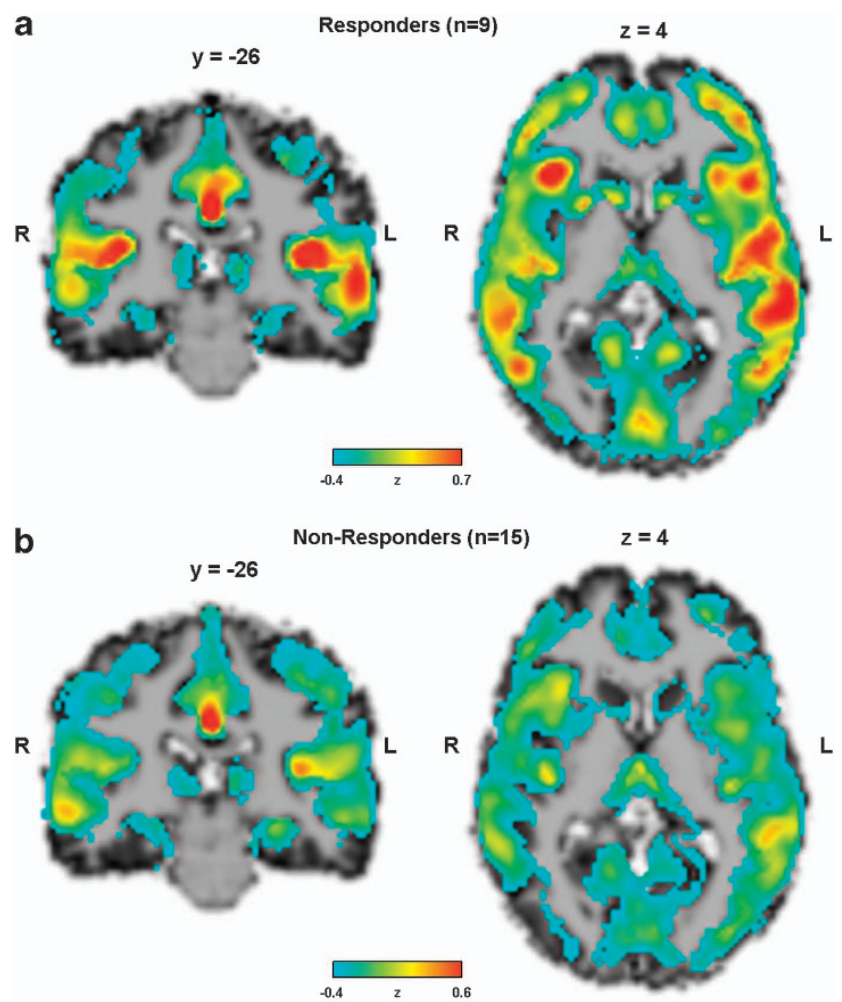

Figure 1 Average mean global CBF images of responders (top) and nonresponders (bottom). CBF values were $z$-transformed.

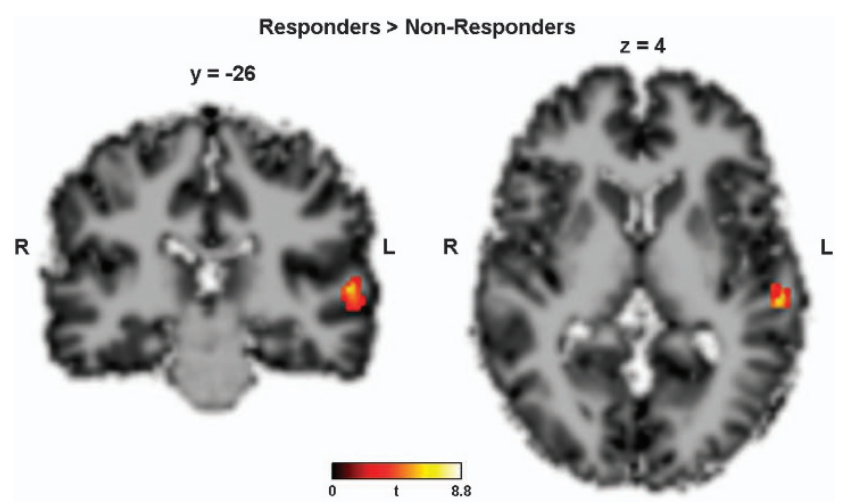

Figure 2 Contrast responders $>$ nonresponders, that is,voxels that were significantly higher perfused in responders than in nonresponders before treatment. Single cluster of 120 voxels survived a cluster-level whole-brain correction for familywise error in the left superior temporal gyrus (Brodmann area 22, peak: $\mathrm{t}[22]=7.28, \mathrm{x} / \mathrm{y} / \mathrm{z}=-58 /-26 / 4)$. No clusters survived in the inverse contrast (nonresponders $>$ responders).

and nonresponders. The area under the ROC curve (AUC) was 0.96 (s.e.: $0.05,95 \%$ confidence interval: $0.87-1.0$, $P<0.0001$, Figure 4). The cutoff point for best sensitivity and specificity was calculated at $z=0.26$ (Figure 4).

\section{Discussion}

Here we could demonstrate that CBF predicts TMS therapeutic response in patients with schizophrenia suffering from $\mathrm{AVH}$. Responders showed a higher resting perfusion in the left

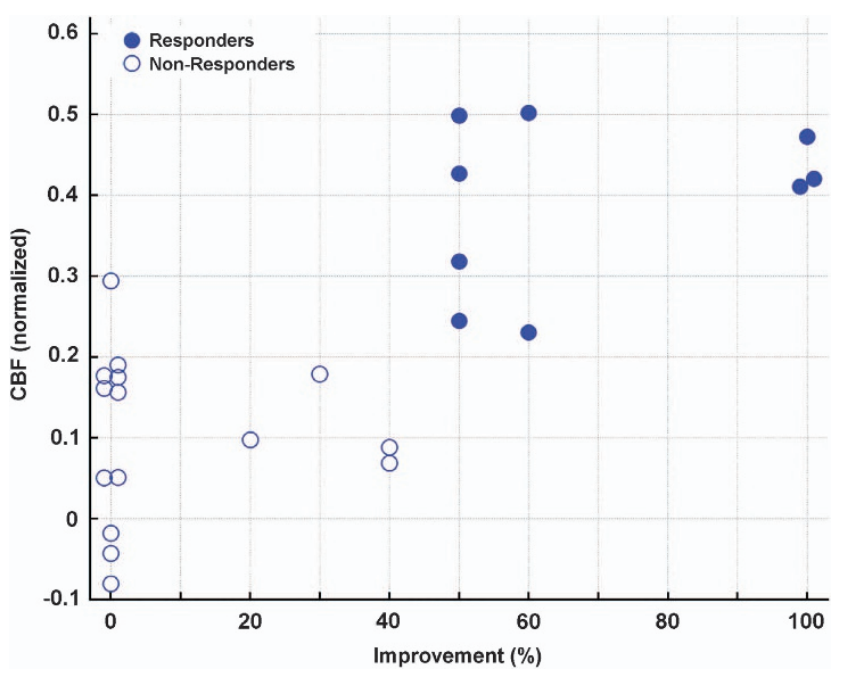

Figure 3 Scatter plot of improvement in percent, as measured with the hallucination change scale and $\mathrm{CBF}$ in the left superior temporal gyrus before treatment (Spearman's rank correlation, $r=0.7$, df: 22, $P<0.0001$ ). CBF values were $z$-transformed.

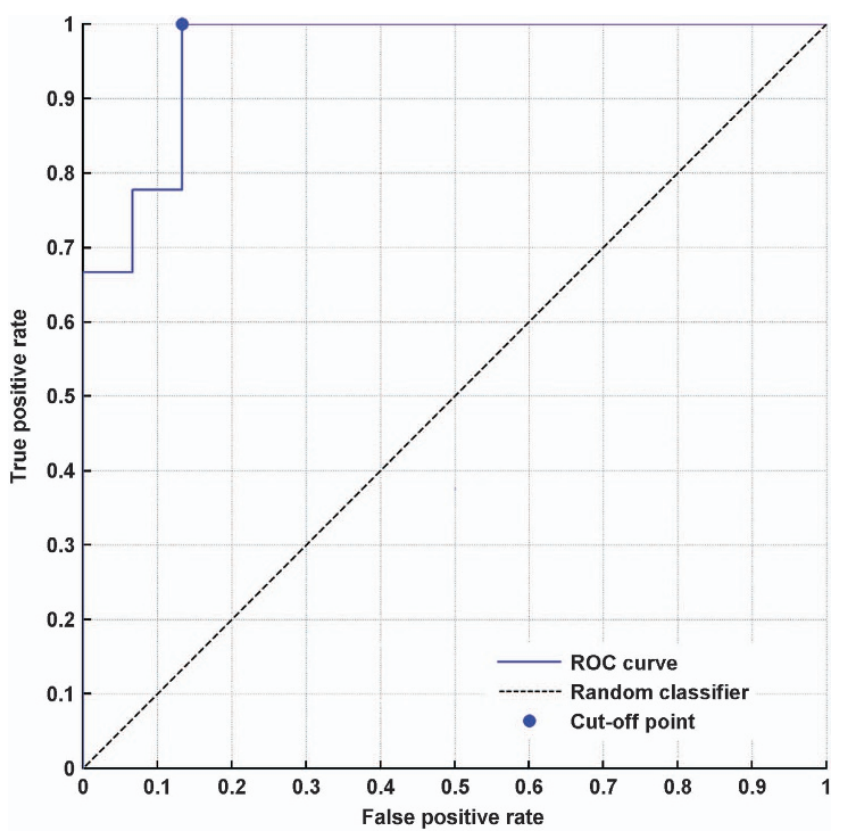

Figure $4 \mathrm{ROC}$ analysis to test the discriminative power of the CBF in the left superior temporal gyrus before treatment in responders and nonresponders to transcranial magnetic stimulation. The AUC was 0.96 (s.e.: $0.05,95 \%$ confidence interval: $0.87-1.0, P<0.0001)$. The cutoff point for best sensitivity and specificity was calculated at $z=0.26$.

STG before treatment. The clinical relevance of this finding derives from the fact that possible responders to TMS might be identified before the treatment by a single noninvasive MR measurement of resting perfusion.

In the present study, a sample of 24 patients with AVH who had been treated for 10 days with TMS was examined to test whether resting perfusion measurement with ASL before treatment could be used to distinguish between responders and nonresponders. A single cluster of 120 voxels in the left 
STG was detected that was significantly higher perfused in responders than in nonresponders and that was very close to the region targeted by TMS, suggesting that the efficacy of TMS depends on the level of brain activation in the area close to the target region. Patients with higher brain activity in the left STG might constitute a specific clinical subgroup of patients responsive to TMS. This is in line with recent findings. The reduced but still significant effect size of TMS on refractory $\mathrm{AVH}$, when compared with previous quantitative reviews that has recently been published, ${ }^{23}$ suggests the possibility that TMS may be effective in clinical subgroups of patients only. In addition, a study with the largest sample size $(n=62)$ so far showed that low-frequency TMS administered to the left temporoparietal cortex or to the site of maximal hallucinatory neuronal activation is not more effective for medication-resistant $\mathrm{AVH}$ than sham treatment. ${ }^{22}$ Although the authors reported that mean severity of $\mathrm{AVH}$ significantly decreased over time, no significant difference in reduction of AVH or any other psychotic symptom was revealed among the three treatment conditions. They described that even when the two groups receiving guided and nonguided TMS were combined ( $n=42)$, TMS was not superior to sham treatment in reducing $\mathrm{AVH}$ or other psychotic symptoms. However, resting brain perfusion was not measured in their study, and thus the missing effect might be explained by lower levels of CBF corresponding to a low neuronal activity in the target region. On the other hand, in the aforementioned study, an online $\mathrm{FMRI}$ paradigm was used during the experience of $\mathrm{AVH}$ to determine the TMS target region. As BOLD signal changes represent relative changes of neuronal activity, they may be associated with unresponsiveness to TMS. Thus, the present study extends the previous ones by suggesting that baseline resting CBF in specific language-related cerebral areas is the key factor determining the efficacy of TMS therapy. More specifically, a nonresponder showing statistically relevant BOLD activations during AVH might still be 'under-perfused' in the same area compared with a responder. Our finding of an elevation in the left STG perfusion in responders to TMS gives further evidence for the involvement of primary language production and perception areas in $\mathrm{AVH}$. It is widely believed that $\mathrm{AVH}$ arise from a disorder of inner verbal experiences and its monitoring. ${ }^{48,49}$ Altered activation in speech production areas (that is inferior frontal gyrus), and altered coupling with monitoring areas (anterior cingulate) and language reception areas (Wernicke's area), has been proposed in a model of bottom-up dysfunction through overactivation in the secondary (and occasionally primary) sensory cortices, which leads to the experience of vivid perceptions in the absence of sensory stimuli. ${ }^{50,51}$ In line with this model, TMS delivered to the left temporoparietal cortex possibly reduces $\mathrm{AVH}^{6,35,52}$ Recently, we published a case of a schizophrenia patient with medication-resistant $\mathrm{AVH}$ that was successfully stimulated at the same region with transcranial direct current stimulation. ${ }^{4}$ These findings suggest that $\mathrm{AVH}$ require the activation of speech perception neurocircuitry, consistent with what has been proposed as a model of underconstrained perception. ${ }^{53}$ Interestingly, none of the covariates demonstrated any prediction power, suggesting that neurobiological factors are more closely related to the possibility of modulating AVH with TMS. In addition, there was no statistical difference between the efficacy of theta-burst and 1-Hz TMS protocols, which is in line with our recent finding that theta burst and $1-\mathrm{Hz}$ TMS are equally effective in the treatment of $\mathrm{AVH} .^{24}$

Several limitations of our study design merit comment. One must consider that no direct relationship between brain activity and the occurrence of hallucinations during scanning was investigated, because in ASL the mean cerebral perfusion over the scanning time is calculated and therefore temporal resolution is low. In addition, these results will have to be replicated in a sham-controlled design that applies the cutoff point found in our study. Both sham and stimulation groups should include an even number of patients with a highenough level of STG perfusion before treatment. Thus, a decision-making algorithm for future studies could be developed following the ROC analysis. A level of normalized CBF in the left STG of at least 0.26 could then be used to decide whether to include or exclude a patient because of expected treatment response in TMS.

How can the higher STG perfusion in responders be explained? Given the fact that the target region for TMS was also situated in the STG, one might speculate that the efficacy of TMS relies on a certain level of brain activity in the area close to the target region. ${ }^{29}$ This is in line with the suggestion that the disturbance of the AVH-generating network by TMS is the key factor. ${ }^{24}$ In the condition of relatively low brain activity in patients, an inhibitive TMS treatment might not be the appropriate treatment approach. Future studies should be aimed at investigating whether there are alternative brain regions in such nonresponders that could be involved in the generation of $\mathrm{AVH}$, and that could be considered the possible target regions for TMS.

In conclusion, this study showed that only patients with higher STG perfusion benefited from TMS as adjuvant treatment. Being noninvasive, relatively easy to use and independent of the occurrence of AVH during scanning, the measurement of resting perfusion with ASL before treatment might be a clinically relevant way to identify possible responders and nonresponders to TMS.

\section{Conflict of interest}

The authors declare no conflict of interest.

Acknowledgements. This work was supported by the Swiss National Science Foundation (SNSF: 32003B-112578 and 33CM30-124114) to TD. We are grateful to A Federspiel and G Schroth for providing the MR-ASL sequence.

1. Sartorius N, Jablensky A, Korten A, Ernberg G, Anker M, Cooper JE et al. Early manifestations and first-contact incidence of schizophrenia in different cultures. A preliminary report on the initial evaluation phase of the WHO collaborative study on determinants of outcome of severe mental disorders. Psychol Med 1986; 16: 909-928.

2. Shergill SS, Murray RM, McGuire PK. Auditory hallucinations: a review of psychological treatments. Schizophr Res 1998; 32: 137-150.

3. Copolov D, Trauer T, Mackinnon A. On the non-significance of internal versus external auditory hallucinations. Schizophr Res 2004; 69: 1-6.

4. Homan P, Kindler J, Federspiel A, Flury R, Hubl D, Hauf M et al. Muting the voice: a case of arterial spin labeling-monitored transcranial direct current stimulation treatment of auditory verbal hallucinations. Am J Psychiatry 2011; 168: 853-854.

5. Hoffman RE, Boutros NN, Berman RM, Roessler E, Belger A, Krystal JH et al. Transcranial magnetic stimulation of left temporoparietal cortex in three patients reporting hallucinated 'voices'. Biol Psychiatry 1999; 46: 130-132. 
6. Hoffman RE, Boutros NN, Hu S, Berman RM, Krystal JH, Charney DS. Transcranial magnetic stimulation and auditory hallucinations in schizophrenia. Lancet 2000; 355 1073-1075.

7. Saba G, Verdon CM, Kalalou K, Rocamora JF, Dumortier G, Benadhira R et al. Transcranial magnetic stimulation in the treatment of schizophrenic symptoms: a double blind sham controlled study. J Psychiatr Res 2006; 40: 147-152.

8. Vercammen A, Knegtering H, Bruggeman R, Westenbroek HM, Jenner JA, Slooff CJ et al. Effects of bilateral repetitive transcranial magnetic stimulation on treatment resistant auditory-verbal hallucinations in schizophrenia: a randomized controlled trial. Schizophr Res 2009; 114: 172-179.

9. Loo CK, Sainsbury K, Mitchell P, Hadzi-Pavlovic D, Sachdev PS. A sham-controlled trial of left and right temporal rTMS for the treatment of auditory hallucinations. Psychol Med 2010; 40: $541-546$.

10. Rosa MO, Gattaz WF, Rosa MA, Rumi DO, Tavares H, Myczkowski M et al. Effects of repetitive transcranial magnetic stimulation on auditory hallucinations refractory to clozapine. J Clin Psychiatry 2007; 68: 1528-1532.

11. Chibbaro G, Daniele M, Alagona G, Di Pasquale C, Cannavo M, Rapisarda V et al. Repetitive transcranial magnetic stimulation in schizophrenic patients reporting auditory hallucinations. Neurosci Lett 2005; 383: 54-57.

12. Brunelin J, Poulet E, Bediou B, Kallel L, Dalery J, D'Amato T et al. Low frequency repetitive transcranial magnetic stimulation improves source monitoring deficit in hallucinating patients with schizophrenia. Schizophr Res 2006; 81: 41-45.

13. Lee SH, Kim W, Chung YC, Jung KH, Bahk WM, Jun TY et al. A double blind study showing that two weeks of daily repetitive TMS over the left or right temporoparietal cortex reduces symptoms in patients with schizophrenia who are having treatment-refractory auditory hallucinations. Neurosci Lett 2005; 376: 177-181.

14. Hoffman RE, Gueorguieva R, Hawkins KA, Varanko M, Boutros NN, Wu YT et al. Temporoparietal transcranial magnetic stimulation for auditory hallucinations: safety, efficacy and moderators in a fifty patient sample. Biol Psychiatry 2005; 58: 97-104.

15. Hoffman RE, Hampson M, Wu K, Anderson AW, Gore JC, Buchanan RJ et al. Probing the pathophysiology of auditory/verbal hallucinations by combining functional magnetic resonance imaging and transcranial magnetic stimulation. Cereb Cortex 2007; 17: 2733-2743.

16. Poulet E, Brunelin J, Bediou B, Bation R, Forgeard L, Dalery J et al. Slow transcranial magnetic stimulation can rapidly reduce resistant auditory hallucinations in schizophrenia. Biol Psychiatry 2005; 57: 188-191.

17. McIntosh AM, Semple D, Tasker K, Harrison LK, Owens DG, Johnstone EC et al. Transcranial magnetic stimulation for auditory hallucinations in schizophrenia. Psychiatry Res 2004; 127: 9-17.

18. Jandl M, Steyer J, Weber M, Linden DE, Rothmeier J, Maurer $\mathrm{K}$ et al. Treating auditory hallucinations by transcranial magnetic stimulation: a randomized controlled cross-over trial. Neuropsychobiology 2006; 53: 63-69.

19. Schonfeldt-Lecuona C, Gron G, Walter H, Buchler N, Wunderlich A, Spitzer M et al. Stereotaxic rTMS for the treatment of auditory hallucinations in schizophrenia. Neuroreport 2004; 15: 1669-1673

20. Fitzgerald PB, Benitez J, Daskalakis JZ, Brown TL, Marston NA, de Castella A et al. A double-blind sham-controlled trial of repetitive transcranial magnetic stimulation in the treatment of refractory auditory hallucinations. J Clin Psychopharmacol 2005; 25: 358-362.

21. Sommer IE, de Weijer AD, Daalman K, Neggers SF, Somers M, Kahn RS et al. Can fMRIguidance improve the efficacy of rTMS treatment for auditory verbal hallucinations? Schizophr Res 2007; 93: 406-408.

22. Slotema CW, Blom JD, de Weijer AD, Diederen KM, Goekoop R, Looijestijn J et al. Can low-frequency repetitive transcranial magnetic stimulation really relieve medicationresistant auditory verbal hallucinations? Negative results from a large randomized controlled trial. Biol Psychiatry 2011; 69: 450-456.

23. Demeulemeester $M$, Amad A, Bubrovszky M, Pins $D$, Thomas $P$, Jardri $R$. What is the real effect of $1-\mathrm{Hz}$ repetitive transcranial magnetic stimulation on hallucinations? Controlling for publication bias in neuromodulation trials. Biol Psychiatry 2012; 71: e15-e16.

24. Kindler J, Homan P, Jann K, Federspiel A, Flury R, Hauf M et al. Reduced neuronal activity in language-related regions after transcranial magnetic stimulation therapy for auditory verbal hallucinations. Biol Psychiatry; e-pub ahead of print 26 July 2012; doi:10.1016/ j.biopsych.2012.06.019

25. Liddle PF, Friston KJ, Frith CD, Hirsch SR, Jones T, Frackowiak RS. Patterns of cerebral blood flow in schizophrenia. Br J Psychiatry 1992; 160: 179-186.

26. Gur RE, Mozley PD, Resnick SM, Mozley LH, Shtasel DL, Gallacher F et al. Resting cerebral glucose metabolism in first-episode and previously treated patients with schizophrenia relates to clinical features. Arch Gen Psychiatry 1995; 52: 657-667.

27. Lahti AC, Weiler MA, Holcomb HH, Tamminga CA, Carpenter WT, McMahon R Correlations between $\mathrm{rCBF}$ and symptoms in two independent cohorts of drug-free patients with schizophrenia. Neuropsychopharmacology 2006; 31: 221-230.

28. Huang YZ, Edwards MJ, Rounis E, Bhatia KP, Rothwell JC. Theta burst stimulation of the human motor cortex. Neuron 2005; 45: 201-206.

29. Nyffeler T, Cazzoli D, Hess CW, Muri RM. One session of repeated parietal theta burst stimulation trains induces long-lasting improvement of visual neglect. Stroke 2009; 40 : 2791-2796.
30. Kindler J, Schumacher R, Cazzoli D, Gutbrod K, Koenig M, Nyffeler T et al. Theta burst stimulation over the right broca's homologue induces improvement of naming in aphasic patients. Stroke 2012; 43: 2175-2179.

31. Viviani R, Sim EJ, Lo H, Beschoner P, Osterfeld N, Maier C et al. Baseline brain perfusion and the serotonin transporter promoter polymorphism. Biol Psychiatry 2010; 67: 317-322.

32. Jann K, Koenig T, Dierks T, Boesch C, Federspiel A. Association of individual resting state EEG alpha frequency and cerebral blood flow. Neuroimage 51: 365-372.

33. Horn H, Federspiel A, Wirth M, Muller TJ, Wiest R, Wang JJ et al. Structural and metabolic changes in language areas linked to formal thought disorder. Br J Psychiatry 2009; 194: $130-138$.

34. Xu G, Rowley HA, Wu G, Alsop DC, Shankaranarayanan A, Dowling M et al. Reliability and precision of pseudo-continuous arterial spin labeling perfusion MRI on 3.0 T and comparison with 150-water PET in elderly subjects at risk for Alzheimer's disease. NMR Biomed 2010; 23: 286-293.

35. Hoffman RE, Hawkins KA, Gueorguieva R, Boutros NN, Rachid F, Carroll K et al. Transcranial magnetic stimulation of left temporoparietal cortex and medication-resistant auditory hallucinations. Arch Gen Psychiatry 2003; 60: 49-56.

36. Kay SR, Opler LA, Lindenmayer JP. Reliability and validity of the positive and negative syndrome scale for schizophrenics. Psychiatry Res 1988; 23: 99-110.

37. Oldfield RC. The assessment and analysis of handedness: the Edinburgh inventory. Neuropsychologia 1971; 9: 97-113.

38. Wang J, Rao H, Wetmore GS, Furlan PM, Korczykowski M, Dinges DF et al. Perfusion functional MRI reveals cerebral blood flow pattern under psychological stress. Proc Natl Acad Sci USA 2005; 102: 17804-17809.

39. Homan P, Kindler J, Hubl D, Dierks T. Auditory verbal hallucinations: imaging, analysis, and intervention. Eur Arch Psychiatry Clin Neurosci 2012; e-pub ahead of print 2 September 2012; doi:10.1007/s00406-012-0355-2.

40. Brett M, Anton JL, Valabregue R, Poline JB. Region of interest analysis using an SPM toolbox. 8th International Conference on Functional Mapping of the Human Brain, vol. 16: Sendai, Japan, 2002.

41. Federspiel A, Muller TJ, Horn H, Kiefer C, Strik WK. Comparison of spatial and temporal pattern for fMRI obtained with BOLD and arterial spin labeling. J Neural Transm 2006; 113 1403-1415.

42. Hickok G, Poeppel D. The cortical organization of speech processing. Nat Rev Neurosci 2007; 8: 393-402

43. Hickok G, Okada K, Serences JT. Area Spt in the human planum temporale supports sensory-motor integration for speech processing. J Neurophysiol 2009; 101 2725-2732.

44. Hashimoto R, Lee K, Preus A, McCarley RW, Wible CG. An fMRI study of functional abnormalities in the verbal working memory system and the relationship to clinical symptoms in chronic schizophrenia. Cereb Cortex 2010; 20: 46-60.

45. Schutter DJ, van Honk JA. Standardized motor threshold estimation procedure for transcranial magnetic stimulation research. J ECT 2006; 22: 176-178.

46. Rossi S, Hallett M, Rossini PM, Safety Pascual-Leone A.. Ethical considerations, and application guidelines for the use of transcranial magnetic stimulation in clinical practice and research. Clin Neurophysiol 2009; 120: 2008-2039.

47. Sack AT, Cohen Kadosh R, Schuhmann T, Moerel M, Walsh V, Goebel R. Optimizing functional accuracy of TMS in cognitive studies: a comparison of methods. J Cogn Neurosci 2009; 21: 207-221.

48. David AS. The neuropsychological origin of auditory hallucinations. In: David AS, Cutting JC (eds). Neuropsychology of Schizophrenia. Erlbaum: Hove, UK, 1994 pp 269-313.

49. McGuire PK, Silbersweig DA, Wright I, Murray RM, David AS, Frackowiak RS et al. Abnormal monitoring of inner speech: a physiological basis for auditory hallucinations. Lancet 1995; 346: 596-600.

50. Hubl D, Koenig T, Strik W, Federspiel A, Kreis R, Boesch C et al. Pathways that make voices: white matter changes in auditory hallucinations. Arch Gen Psychiatry 2004; 61: 658-668.

51. Hubl D, Koenig T, Strik WK, Garcia LM, Dierks T. Competition for neuronal resources: how hallucinations make themselves heard. Br J Psychiatry 2007; 190: 57-62.

52. Aleman A, Sommer IE, Kahn RS. Efficacy of slow repetitive transcranial magnetic stimulation in the treatment of resistant auditory hallucinations in schizophrenia: a metaanalysis. J Clin Psychiatry 2007; 68: 416-421.

53. Behrendt RP, Young C. Hallucinations in schizophrenia, sensory impairment, and brain disease: a unifying model. Behav Brain Sci 2004; 27: 771-787; discussion 787-830.

Translational Psychiatry is an open-access journal published by Nature Publishing Group. This work is licensed under the Creative Commons Attribution-NonCommercial-No Derivative Works 3.0 Unported License. To view a copy of this license, visit http://creativecommons.org/licenses/by-nc-nd/3.0/ 